\title{
TEM Investigation of Deformation Mechanisms in FeMnCrCN TWIP Steel
}

\author{
J.E. Wittig ${ }^{1}$, D. T. Pierce ${ }^{1}$, L. Mosecker ${ }^{2}$, A. Saeed-Akbari ${ }^{3}$, M. Beigmohamadi ${ }^{4}$, J. Mayer ${ }^{4}$ \\ 1. Interdisciplinary Materials Science, Vanderbilt University, Nashville TN, USA \\ 2. Institute for Eisenhüttenkunde, RWTH University, Aachen, Germany \\ 3. Department of Materials, ETH Zurich, Zurich, Switzerland \\ 4. Gemeinschaftslabor für Elektronenmikroskopie, RWTH University, Aachen, Germany
}

A new class of austenitic steels with high-Mn content exhibits exceptional mechanical properties, such as large energy absorption and high work-hardening rate, owing to a mechanical twinning induced plasticity (TWIP) deformation mechanism [1]. The replacement of Ni with $\mathrm{Mn}$ as an austenite stabilizer decreases the stacking-fault energy (SFE) in the steel, which favors mechanical twinning over dislocation glide. The interaction of perfect and partial dislocations with twin boundaries during mechanical deformation enhances the work hardening, i.e., a dynamic Hall-Petch effect. In this investigation, an Fe-Mn austenitic stainless steel has been studied using weak-beam dark field (WBDF) imaging of partial dislocations for SFE measurement, bright field (BF) imaging of the deformation mechanisms, and high-resolution imaging (HRTEM) of the twinning interface to better understand the mechanical deformation and work hardening mechanism.

The austenitic stainless steel with nominal composition, Fe-16Mn-14Cr-0.3C-0.3N (wt\%), was strip cast, cold rolled to a final thickness of $1.1 \mathrm{~mm}$, and recrystallized. The material was fully austenitic in the as-received state with an average grain size of $\sim 20 \mu \mathrm{m}$. Samples deformed in tension at a strain rate of $10^{-3} \mathrm{~s}^{-1}$ to elongations of $1.5 \%$ and $20 \%$ were prepared for TEM by standard twin-jet electropolishing in $70 \%$ methanol $30 \%$ perchloric acid at $20 \mathrm{~V}$ and $-30^{\circ} \mathrm{C}$. These samples were analyzed using a Philips CM20, FEI Tecnai F20, and an image-corrected FEI Titan 80-300. The atomic-resolution imaging was facilitated by first using the Philips CM20 to identify grains with minimal bend contours and a $<110>$ zone axis that contained the (111) twin plane close to the flat position prior to imaging with the Titan.

The WBDF image in Figure 1 shows an example of partial dislocations from a sample strained to $1.5 \%$ where the average separation distance is $9.2+/-1.2 \mathrm{~nm}$. The sample was oriented with a beam direction B near [111] which simplifies the partial separation measurements and Burgers vector analysis. With B near [111], a geometrical conversion of the observed separation to the actual separation due to inclination is not necessary for dislocations that lie on the (111) glide plane [2]. Figure 2 is a graph of partial dislocation separation vs. the dislocation character angle (B). The experimental best-fit of SFE $(\gamma)$, which is related to the partial-dislocation separation $\left(\mathrm{d}_{\text {actual }}\right)$ by the relationship [3] in figure 2 , is 22 $+/-4 \mathrm{~mJ} \mathrm{~m}^{-2}$, where $\mu$ is the shear modulus $(77.8 \mathrm{GPa}), \mathrm{b}_{\mathrm{p}}$ is the partial-dislocation Burgers vector $(0.146 \mathrm{~nm})$, and $v$ is the Poisson ratio (0.292).

The BF image in figure 3 from a sample strained to $20 \%$ elongation reveals that this value of SFE correlates with TWIP deformation behavior. This two-beam image, produced using the twin plane gvector $(\mathbf{g}=111)$, shows mechanical twins ranging from 10 to $40 \mathrm{~nm}$ in width. The twin boundaries are effective obstacles for dislocation slip and the partial dislocations from extended stacking faults terminate at the twin interfaces. Although the sample thickness is greater than ideal for HRTEM, Figure 4 is a high-resolution image from the area of figure 3 with a twin less than $5 \mathrm{~nm}$ wide. Other HRTEM images from this area revealed twins of only 3 or 4 atomic layers. The strain at the twin boundaries is evident from the disturbances in the image quality. However, specimen mis-alignment and sample thickness could also contribute to these effects. These HRTEM images, which are some of the first from these TWIP materials, offer the opportunity to investigate the strain fields at twin boundary/dislocation interactions using the geometric-phase technique from the filtered inverse Fourier transform of the HRTEM images [4]. 


\section{References}

[1] O. Grassel, L. Kruger, G. Frommeyer, and L. W. Meyer, Int. J. Plasticity, vol. 16 (2000) p. 1391

[2] D. T. Pierce, J. Bentley, J. A. Jimenez and J. E. Wittig, Scripta Mat. 66 (2012) p. 753

[3] E. Aerts, P. Delavignette, R. Siems, and S. Amelinckx, J. Appl. Phys., vol. 33, no. 10, (1962) p. 3078 [4] Financial support from the NSF DMR 0805295 and the DFG SFB 761 is gratefully acknowledged.

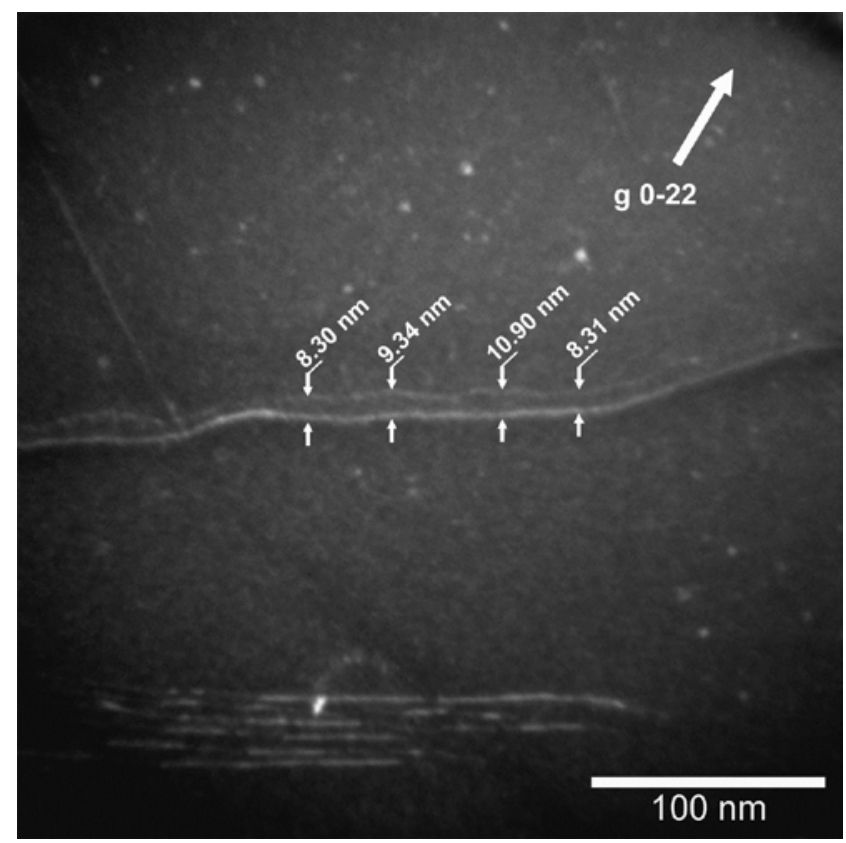

Figure 1. WBDF image of partial dislocations with $g=3 g(220)$.

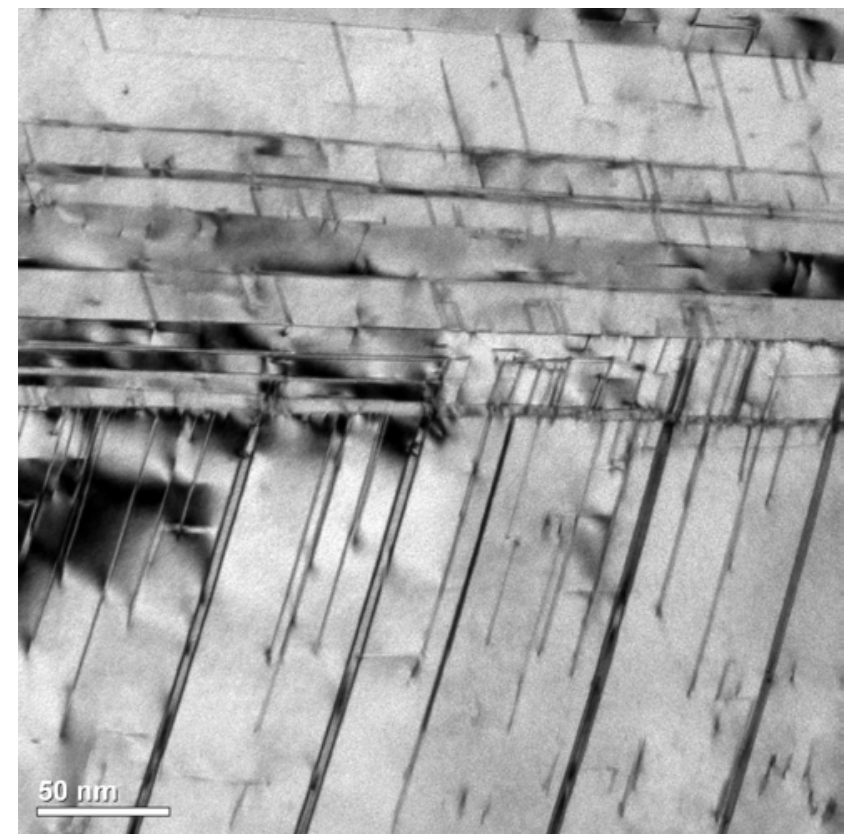

Figure 3. $\mathrm{BF}$ image of twinning and extended stacking faults, $20 \%$ elongation.

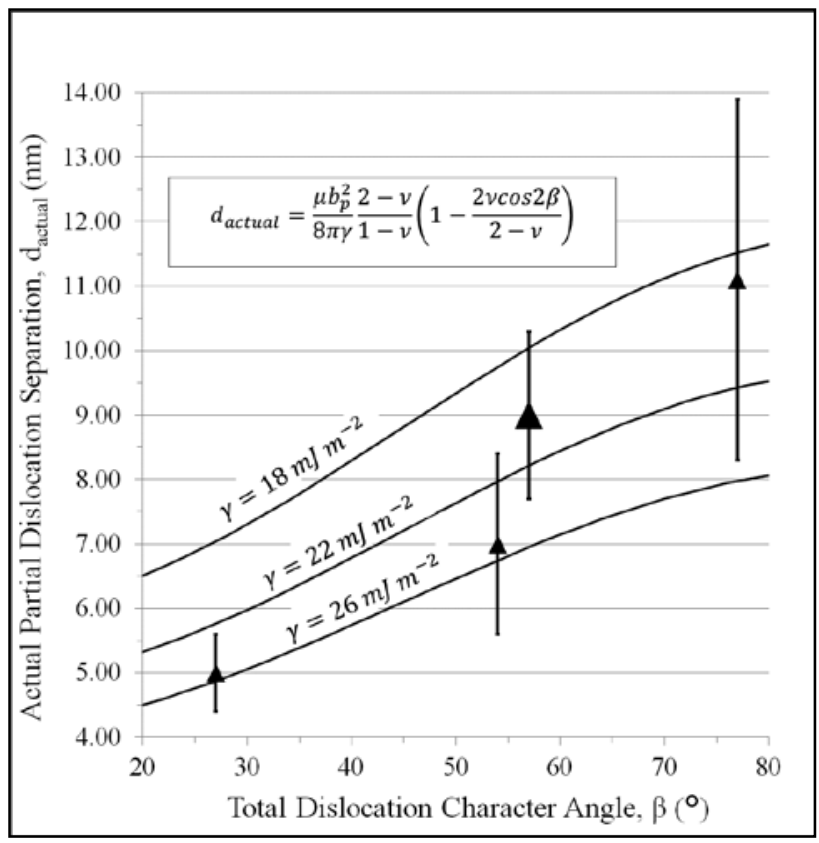

Figure 2. Partial Dislocation Separation vs Character Angle.

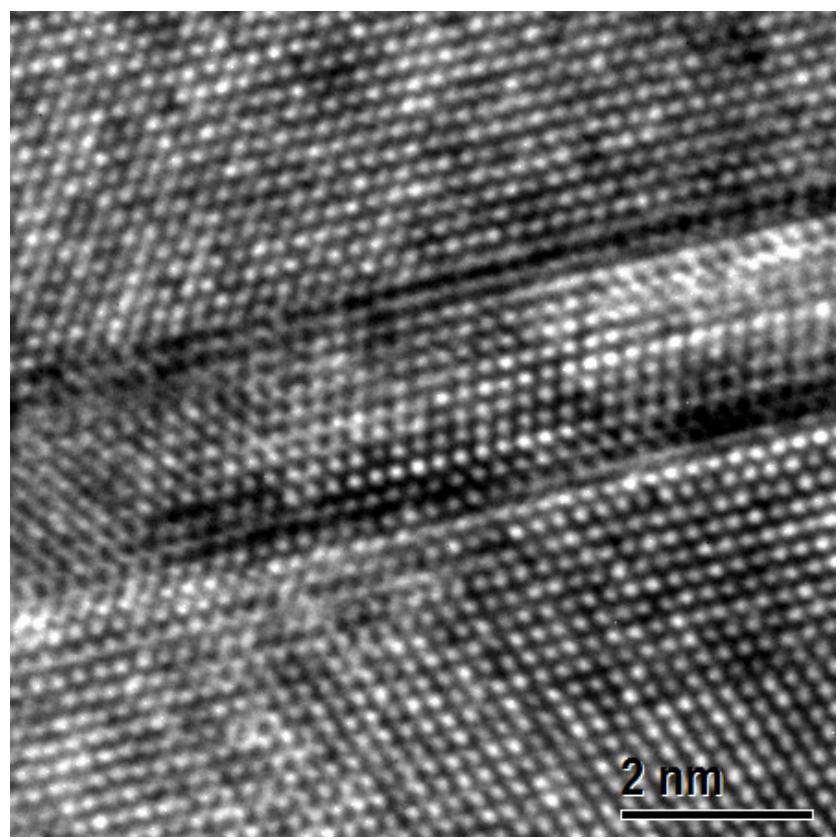

Figure 4. HRTEM image from area in figure 3. 